Referencia para citar este artículo: Ruiz-Ramírez, R.; Sánchez-Romero, C.; Zapata-Martelo, E.; García-Cué, J. L.; Pérez-Olvera, Ma. A.; Martínez-Corona, B. \& Rojo-Martínez, G. (2017). Manifestaciones del bullying en la Preparatoria Agrícola. Universidad Autónoma de Chapingo, México. Revista Latinoamericana de Ciencias Sociales, Niñez y Juventud, 15 (2), pp. 1149-1163. DOI:10.11600/1692715x.1522425112016

\title{
Manifestaciones del bullying en la Preparatoria Agrícola. Universidad Autónoma de Chapingo, México*
}

\author{
ROSALVA RUIZ-RAMÍREZ ** \\ Estudiante Colegio de Postgraduados, México. \\ CRISTINA SÁNCHEZ-ROMERo ${ }^{* * *}$ \\ Profesora Universidad Nacional de Educación a Distancia, España.

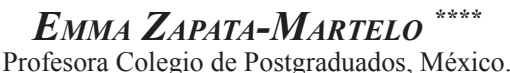 \\ JoSÉ LUIS GARCÍA-CUÉ *****
Profesor Colegio de Postgraduados, México.

\section{Ma. Antonia PÉREZ-OlVERA ${ }^{* * * * * * *}$} \\ Profesora Colegio de Postgraduados, México.

\section{BEATRIZ MARTÍNEZ-CORONA ${ }^{* * * * * *}$} \\ Profesora Colegio de Postgraduados, México. \\ GUSTAVO RoJo-MaRTínez \\ Profesor Universidad Autónoma Indígena de México, México.
}

\footnotetext{
* $\quad$ Este artículo de investigación científica y tecnológica se deriva de un estudio de mayor escala denominado "Bullying en escuelas rurales del nivel medio superior", forma parte de una investigación de Doctorado en Ciencias en el área de Desarrollo rural en México. La entidad que avaló la investigación fue el Colegio de Postgraduados, México. Inició en enero de 2015 y finalizó en diciembre de 2015. Para correspondencia: jlgcue@colpos.mx. Área: Ciencias de la educación; Subárea: educación general; problemas educativos.

** Maestra en Ciencias en Desarrollo Rural, por Colegio de Postgraduados, Maestra en Ciencias en Educación Humanista por el Instituto Humanista de Sinaloa, Licenciada en Biología por el Instituto Tecnológico de Los Mochis. Orcid: 0000-0002-0845-5945. Índice H5: 1. México. Correo electrónico: rosalva.ruiz@colpos.mx

*** Doctora por la Universidad Nacional de Educación a Distancia. Master en Informática Educativa. Licenciada en Filosofía y Ciencias de la Educación. España. Orcid: 0000-0002-0280-5406. Índice H5: 8. Correo electrónico: csanchez@edu.uned.es

**** $\quad$ Maestra y Doctora en Ciencias en Sociología por la Universidad de Texas, Estados Unidos. Orcid: 0000-0002-1623-3322. Índice H5: 12. Correo electrónico: emzapata@colpos.mx

***** Doctor en Ciencias en Educación por la Universidad Nacional de Educación a Distancia, España. Maestro en Ciencias en Computo Aplicado, por Colegio de Postgraduados e Ingeniero Mecánico Electricista en Universidad La Salle. México. Orcid: 0000-0001-6367-2339. Índice H5: 7. Correo electrónico: jlgcue@colpos.mx

****** Doctora en Ciencias por la Universidad Autónoma Chapingo. Maestra en Ciencias en Edafología, por Colegio de Postgraduados e Ingeniera Agrónoma Fitotecnista, por Universidad Autónoma Chapingo. México. Orcid: 0000-0002-6408-8641. Índice H5: 6. Correo electrónico: molvera@colpos.mx

******* Doctora en Ciencias en Estrategias de Desarrollo Agrícola Regional por Colegio de Postgraduados, Maestra en Ciencias en Desarrollo Rural, Lic. En Psicología por la Universidad Nacional Autónoma de México. Orcid: 0000-0002-0745-4270. Índice H5: 9. Correo electrónico: beatrizm@colpos.mx

Doctor en Ciencias en Forestal por el Colegio de Postgraduados, Maestro en Ciencias Forestales por Universidad Autónoma Chapingo, Ingeniero Agrícola por la Universidad Nacional Autónoma de México. Orcid: 0000-0001-8739-6105. Índice H5: 6. Correo electrónico: grojomtz@yahoo.com.mx
} 


\section{Artículo recibido en noviembre 25 de 2016, artículo aceptado en febrero 22 de 2017 (Eds.)}

- Resumen (analítico): el objetivo de la presente investigación fue analizar las manifestaciones del bullying que los estudiantes reciben, hacen u observan en la Preparatoria Agrícola de la Universidad Autónoma de Chapingo ubicada en el Estado de México, México. La metodología fue de corte cuantitativo. Se aplicó un cuestionario a una muestra de 112 estudiantes (43 mujeres y 69 hombres). La información se analizó a través de estadísticos descriptivos univariados y análisis de correlación de Sperman. Los resultados muestran que los estudiantes han sido víctimas de manifestaciones de bullying de tipo psicológicas, verbales, físicas, en redes cibernéticas y sociales. Las víctimas, acosadores y acosadoras consideran que la principal manifestación del bullying que reciben o hacen es psicológica (ignoran o son ignoradas e ignorados); observadores y observadoras especifican que principalmente ven agresiones fisicas (empujones).

Palabras clave: acoso escolar, relaciones entre pares, agresión, escuela de agricultura (Tesauro de Ciencias Sociales de la Unesco).

\section{Acts of bullying in an agricultural high school-Autonomous University of Chapingo, Mexico}

- Abstract (analytical): this research analyzes the acts of bullying that students receive, perpetrate or observe among their peers. The study was carried out in an agricultural school in the Municipality of Texcoco, State of Mexico. The research employed a qualitative methodology with a sample size of 112 students (43 females and 69 males). A questionnaire was used to collect data, the data was analyzed through univariate descriptive statistics and Spearman's correlation analysis. The results detail how students have been bullied by their peers through psychological, verbal, physical, cyber and social attacks. Victims and their bullies consider that the main form of bullying they receive or carry out is psychological (they are ignored or they ignore someone). The students who observe bullying specify that they mainly see physical aggression (pushing).

Key words: school bullying, peer relationships, aggression, agricultural schools (Social Science Unesco Thesaurus).

\section{Manifestações de bullying na preparação agrícola. Universidade Autônoma Chapingo, México}

- Resumo (analítico): o objetivo da pesquisa foi analisar as manifestações do bullying que os estudantes recebem ou fazem na preparação agrícola da Universidade Autônoma de Chapingo situada no Estado do México, México. A metodologia foi de caráter quantitativo. Aplicou-se um questionário a 112 estudantes (43 mulheres e 69 homens). A informação foi analisada através de estatística descritiva e análise de correlação de Sperman. Os resultados mostram que os estudantes têm sido vítimas de manifestações de bullying dos tipos psicológicos, verbais, físicos, em redes cibernéticas e sociais. As vítimas e os acusados como consideram que a principal manifestação do bullying que recebem ou fazem é a psicológica (ignoram ou são ignoradas); Observadores e observadoras especificam principalmente agressões físicas (empurrões).

Palavras-chave: abuso escolar, relações entre pares, agressão, escola de agricultura (Thesaurus de Ciências Sociais da Unesco).

\section{-1. Introducción. -2. Metodología. -3. Resultados y discusiones. -4. Conclusiones. -Lista de referencias.}

\section{Introducción}

El bullying es un problema que obstaculiza el desarrollo del proceso de enseñanza y aprendizaje en las instituciones escolares de México, ya que desencadena diversos problemas como deserción escolar, reprobación, bajo rendimiento académico, ausentismo, entre 
otros, que impactan negativamente en los logros educativos (Del Tronco, 2013; Mendoza, 2012). El bullying se define como un tipo de violencia que el alumno o la alumna sufre o ejerce, el cual, se repite y prolonga; se produce en una situación de desigualdad, y persiste por la ignorancia o pasividad de quienes rodean a los agresores o agresoras y a las víctimas (DíazAguado, 2005).

La Encuesta Nacional sobre Exclusión, Intolerancia y Violencia (Subsecretaría de Educación Media Superior-Sems, 2014) determinó que en el nivel Medio Superior 72\% de los hombres y $65 \%$ de las mujeres han sido víctimas de las diferentes manifestaciones de bullying.

Ante esas cifras, el bullying es un tema prioritario por investigar debido a sus graves consecuencias (Ruiz-Ramírez, Zapata-Martelo, García-Cué, Pérez-Olvera, Martínez-Corona \& Rojo-Martínez, 2016). En ese sentido, desde el año 2014, Senadores/as, Diputados/ as, autoridades de la Secretaria de Educación Pública, entre otros, han propuesto foros, programas educativos y reformas de leyes municipales, estatales y nacionales para prevenir, atender, mitigary sancionar el bullying. Sin embargo, las acciones implementadas no han cumplido los objetivos, debido a que el bullying se ha incrementado en los diferentes niveles y contextos escolares (Zapata-Martelo \& Ruiz-Ramírez, 2015).

En una situación de bullying existen tres actores/actrices principales: acosadores/as, víctimas y observadores/as denominado como "triángulo del bullying" (Mendoza, 2012; Castro, 2006; Díaz-Aguado, 2005).

1. Victima: Es la persona que sufre la agresión, por lo general es un estudiante tímido/a, inseguro/a, con poca fuerza física, talla baja, con obesidad, problemas de lenguaje, pobres habilidades sociales, ansiosos/as, vulnerables, con pocos o ningún amigo/a (Delgado de los Santos, 2015; Brunstein, Marrocco, Kleiman, Schonfeld \& Gould, 2007; Tamar, 2005). Presentan dificultades de aprendizaje o son inteligentes. El riesgo de ser víctimas se incrementa al pertenecer a una cultura étnica (Varhama \& Björkqvist, 2005; Tamar, 2005). Smith, Rigby y Pepler (2004) diferencian dos tipos de víctimas:
- Pasivas, son estudiantes inseguros/as que no responden a los ataques e insultos del agresor/a, sufren calladamente las agresiones, son aislados/as, carecen de asertividad y baja autoestima, además, manifiestan vulnerabilidad al no defenderse de la intimidación.

- Activas, son discentes que se caracterizan por estimular la ira de sus agresores/ as, para su entorno resultan irritantes, impulsivos/as, agresivos/as e incomodos/ as lo que provoca el rechazo de sus compañeros/as; se les considera como "provocadores/as".

2. Acosador o acosadora: Ruiz-Ramírez et al. (2016), Delgado de los Santos (2015), Ghiso (2012), Castro (2006) coinciden que el alumnado que ejerce la agresión sobre las víctimas son violentos/as, abusan de su fuerza, impulsivos/as, con escasas habilidades sociales, baja tolerancia a la frustración, baja autoestima, no cumplen normas; tienen relaciones negativas y bajo rendimiento académico, no son autocríticos/as, necesitan dominar; no son empáticos/as, no se arrepienten; dependiendo de su personalidad puede ser intelectual y utilizar su fama para obligar a sus víctimas a obedecer sus órdenes o puede ser poco inteligente.

Se destacan dos clasificaciones de acosadores/as o agresores/as, la primera de Olweus (1999) que define tres distintos tipos: los activos/as quienes maltratan personalmente y establecen relaciones directas con las víctimas; los sociales-indirectos que dirigen el comportamiento de sus seguidores/ as induciéndolos/as a actos de violencia y persecución; y los agresores/as pasivos/as que son seguidores de los activos/as y no toman la iniciativa en las agresiones. La segunda de Delgado de los Santos (2015), quien también propone tres grupos: los intelectuales, quienes utilizan su fama en el medio social para obligar a sus víctimas a que obedezcan sus órdenes; los poco inteligentes, que se distinguen por ser personas solitarias, acosan a sus víctimas directamente a consecuencia de su baja autoestima; y las víctimas que son acosados/as por personas mayores a ellos, y a su vez agreden a población de menor edad.

3. Observador $u$ observadora: El espectador/a o testigos/as son compañeros 
o compañeras de los victimarios o víctimas; sólo se limitan a observar las situaciones de agresión. Para Delgado de los Santos (2015) tienen poca sensibilidad, se sienten inseguros/ as e impotentes.

Para Delgado de los Santos (2015), la Secretaría de Seguridad Pública-SSP y la Secretaría de Educación Pública-SEP (2007) las y los observadores desempeñan un papel importante al tener el rol de fomentadores/as o inhibidores/as del bullying, ya que al reírse o aceptar el maltrato contribuyen a perpetuarlo y reforzarlo. Asimismo, su participación para solucionarlo es fundamental, al contener las agresiones sí evitan aplaudirlo o apoyan a las víctimas a denunciar (Hidalgo-Rasmussen \& Hidalgo-San Martín, 2015, SSP \& SEP, 2007).

Los observadores/as se clasifican en: a) Cómplices, son el apoyo principal del agresor/a, guían dando indicaciones para atraer a las víctimas; b) Reforzadores, apoyan las agresiones y motivan a que se susciten continuamente; c) Indiferentes, sólo se limitan a observar y callar, no participan en las agresiones; su silencio es porque lo disfrutan o temen ser las próximas víctimas y d) Defensores/as, apoyan a la víctima, aunque en ocasiones sufren las consecuencias de las agresiones (Delgado de los Santos, 2015).

Olweus (2006) explica que el bullying se manifiesta de manera directa e indirecta. En la primera, el alumnado agrede a sus víctimas de forma física o verbal, se produce como resultado de una intensa activación de emociones negativas, como ira o miedo, lo que impide que el alumnado se exprese con asertividad. En la segunda, coincide con Sullivan, Cleary y Sullivan (2005), se presenta cuando el estudiantado agrede de forma psicológica o social, esta no es percibida entre la comunidad educativa, convirtiéndose en peligrosa.

El bullying se manifiesta de diferentes formas entre iguales mujeres y mujeres, hombres y hombres, o intragéneros, las mujeres utilizan agresiones psicológicas y la exclusión social (Skrzypiec, 2008; Olweus 2006; DíazAguado, 2005); los hombres practican las agresiones físicas y verbales (Skrzypiec, 2008; Defensor del Pueblo, 2007).

Mendoza (2012) ha identificado y clasificado cuatro tipos de manifestaciones del bullying:
- Agresiones físicas: Para Brunstein et al. (2007), Voors (2005), la principal manifestación del bullying es la física. Destacan: patear, empujar, pellizcar, jalar el cabello, aventar objetos, escupir, robar, esconder o romper pertenencias, y amenazar con armas (Delgado de los Santos, 2015; Del Tronco, 2013; Mendoza, 2012; Díaz-Aguado, 2005). Una manifestación física que se ha acrecentado es el acoso sexual, desde manoseo hasta violación sexual (OrtegaRivera, Jiménez \& Ruiz, 2010).

- Agresiones verbales: Se identifican como ofensas que producen daño emocional en quien las recibe, bajan la autoestima y dañan el auto-concepto de las víctimas, quienes interiorizan dichas ofensas. Delgado de los Santos (2015), Del Tronco (2013), Mendoza (2012) establecen las siguientes: amenazar, amedrentar, humillar, denigrar, poner apodos, burlarse e insultar. Para Defensor del Pueblo (2007) la manifestación verbal es la más habitual, por la facilidad de la acción; además es aceptada por la sociedad considerándose "normal" (Del Tronco, 2013). Los hombres usan más la agresión verbal que las mujeres (Defensor del Pueblo, 2007).

- Agresiones psicológicas: Son actitudes interpersonales que dañan la autoestima de las víctimas y fomentan la sensación de temor (Del Tronco, 2013; Mendoza, 2012). De acuerdo con Olweus (2006) está agresión es la más común de bullying indirecto, son las más dañinas al ser difíciles de identificar (Onetto, 2004). Para Del Tronco (2013) entre más tiempo se expongan las víctimas a estas agresiones, el daño es mayor y profundo perjudicando su autoestima y auto-percepción; se consideran conductas como: ignorar, no dejar participar, aislar, desprestigiar o difamar, encerrar en una aula, enviar notas hirientes, obligar a hacer cosas que no quieren.

- Agresiones sociales: Acciones que pretende aislar a las víctimas posicionándolas en un status inferior. Existe discusión sobre lo expuesto 
por Voors (2005), en cuanto a que las agresiones sociales sean innatas en el ser humano; una forma de agresión frecuente, presente en diversas culturas, es la discriminación (García-Villanueva, De la Rosa-Acosta \& Castillo-Valdés, 2012) y la exclusión social, ésta se usa para castigar a quienes no se ajustan a las reglas impuestas por la o el líder, las víctimas son aisladas del grupo, es más común entre mujeres (Olweus, 2006; Díaz-Aguado, 2005).

Después de analizar las diferentes propuestas de los autores/as se considera necesario prevenir, atender y mitigar el bullying en especial en bachilleratos con enfoques agrícolas en México. Por tal razón, se investigó sobre estudios en este tipo de instituciones, se encontraron datos de la Secretaria de Educación Pública (SEP) muy generales sobre el bullying en bachilleratos (Secretaría de Educación Pública-SEP, 2014), no se especifican datos por tipo de institución. Se investigó sobre publicaciones del bullying en México y se distinguieron notas periodísticas con cifras, pero no hay ninguna que muestre el comportamiento de observadores/as, víctimas y acosadores/as, por lo que es necesario profundizar sobre este tema (Ruiz-Ramírez et al., 2016).

Por lo anterior, surge la siguiente pregunta ¿Cuáles son las manifestaciones del bullying que observan, hacen y reciben estudiantes de la Preparatoria Agrícola de la Universidad Autónoma Chapingo (UACh)? Para dar respuesta se planteó como objetivo de esta investigación analizar las manifestaciones del bullying en estudiantes de la Preparatoria Agrícola, en Chapingo, México. El supuesto planteado es que en bachillerato se identifican distintas manifestaciones de bullying en observadores/as, víctimas y acosadores/as.

Para cumplir con la pesquisa se trabajó con discentes de un bachillerato público en ciencias agrícolas ubicado en el Oriente de Texcoco, Estado de México, México. Éste alberga estudiantes de diferentes estados del país, algunos pertenecen a distintos grupos indígenas, culturas, creencias, orientaciones, niveles económicos o sociales, entre otros. Las y los estudiantes provienen, por lo general, de comunidades rurales y buscan la inserción a la Preparatoria Agrícola por ser pública y por percibir una beca. El tipo de beca depende del puntaje obtenido en su examen de admisión, de un estudio socioeconómico y del lugar de procedencia. Existen tres tipos de estudiantes; 1) becados internos, incluye hospedaje, alimentos y servicios dentro de las instalaciones de la Universidad; 2) becados externos, consiste en apoyo económico para hospedaje, pasajes y alimentos (servicio que les da la universidad); y 3) externa, el alumno/a tiene derecho y acceso a los servicios universitarios, regularmente en este grupo se ubican a alumnos/as de las zonas aledañas a la Universidad (Estado de México y Ciudad de México, que viven con su familia). Las tres categorías no pagan inscripción, ni colegiaturas y reciben: atención médica, viáticos para viajes de estudio y apoyo para gestiones administrativas.

En ese sentido, padres y madres de familia incentivan a sus hijos/as para ingresar a la institución, ya que una vez aceptados/as, si aprueban todos sus cursos, tienen cubierta su educación de bachillerato y universidad, lo que es una preocupación menos para las familias rurales. Aunado a lo anterior, la Preparatoria Agrícola y la Universidad son de reconocido prestigio por su excelencia académica, por sus planes de estudios, su infraestructura, el nivel académico del profesorado y de sus egresados/ as.

\section{Metodología}

La investigación fue de tipo cuantitativa, no experimental, descriptiva y correlacional. La población fueron alumnos y alumnas de la Preparatoria Agrícola de la UACh, con matricula vigente en diciembre de 2015, con un total de 3,168 estudiantes. La muestra fue de 112 discentes, 39 de primer semestre (21 hombres y 18 mujeres), 37 de tercer semestre (24 hombres y 13 mujeres) y 36 de quinto semestre (24 hombres y 12 mujeres).

El instrumento de recolección de datos fue un cuestionario de elaboración propia con cuatro secciones: datos personales, información sociodemográfica, relaciones personales y relaciones escolares. Esta última sección se dividió en manifestaciones del bullying considerando las agresiones físicas, verbales, 
psicológicas, sociales y cibernéticas, las cuales pueden recibir las víctimas, hacer los agresores/ as o mirar los observadores/as.

El cuestionario se sometió a revisión de expertos, prueba piloto, validación de contenido y análisis de Fiabilidad Alfa de Cronbach dando un total de 0.815 de un máximo de 1 , lo que expresa que el instrumento es fiable.

La aplicación del cuestionario duró 20 minutos, se hizo de manera presencial en los salones de clase. La información se analizó con el paquete estadístico IBM-SPSS V23, se utilizaron estadísticos descriptivos univariados y análisis de correlación de Sperman.

\section{Resultados y discusiones}

Agresiones que reciben las víctimas de bullying por parte de sus compañeros $y$ compañeras

Los alumnos (22.9\%) de la Preparatoria Agrícola han manifestado ser víctimas de bullying por parte de sus compañeros o compañeras, en contraste con $9 \%$ de las alumnas, es decir, los hombres están más expuestos a ser víctimas del bullying, esto se debe a que en la cultura mexicana, más en la rural, los hombres tienen que mostrar su hombría, fuerza y poder ante sus compañeras y compañeros, por lo que tienden a victimizar a sus homólogos, y así ganar su status de "machos".

En la presente investigación, la Tabla 1 muestra que la manifestación indirecta, las agresiones psicológicas $(59.4 \%)$, fueron las más constantes, en contraste con los estudios de Olweus (2006) y García (2010) quienes manifestaron que la agresión física es la más común; mayormente se presentó me han ignorado, al menos una vez a la semana, en hombres $(26.7 \%)$ y en mujeres $(17.8 \%)$, está agresión se exhibe cuando las compañeras/os no escuchan o no ponen atención a lo que las víctimas comentan, dañando su autopercepción y autoestima, coincidiendo con Del Tronco (2013) y Mendoza (2012). Las víctimas consideran que sus opiniones, pensamientos o comentarios no son válidos, por lo que se quedan callados/as ante cualquier duda o comentario dentro y fuera del aula escolar, perdiendo su valía.

Tabla 1. Manifestaciones del bullying que reciben las víctimas.

\begin{tabular}{|c|c|c|c|c|c|c|c|}
\hline \multirow[b]{2}{*}{ Manifestaciones del bullying } & \multicolumn{2}{|c|}{ Una vez a la semana } & \multicolumn{2}{|c|}{ Dos veces a la semana } & \multicolumn{2}{|c|}{ Diario } & \multirow[b]{2}{*}{$\begin{array}{c}\text { Porcentaje } \\
\text { total }\end{array}$} \\
\hline & $\begin{array}{c}\text { Hombres } \\
\%\end{array}$ & $\begin{array}{c}\text { Mujeres } \\
\%\end{array}$ & $\begin{array}{c}\text { Hombres } \\
\%\end{array}$ & $\begin{array}{c}\text { Mujeres } \\
\%\end{array}$ & $\begin{array}{c}\text { Hombres } \\
\%\end{array}$ & $\begin{array}{c}\text { Mujeres } \\
\%\end{array}$ & \\
\hline \multicolumn{8}{|l|}{ Psicológicas } \\
\hline Te ignoran & 26.7 & 17.8 & 5.3 & 5.3 & 2.6 & 1.7 & 59.4 \\
\hline Te hacen gestos & 13.3 & 12.5 & 5.3 & 1.7 & 0.8 & 1.7 & 35.3 \\
\hline Te difaman & 15.1 & 2.6 & 4.4 & 1.7 & 1.7 & 0 & 25.5 \\
\hline Recibes miradas morbosas & 3.5 & 11.6 & 0.8 & 1.7 & 0.8 & 0.8 & 19.2 \\
\hline \multirow{2}{*}{\multicolumn{8}{|c|}{ Verbales }} \\
\hline & & & & & & & \\
\hline Hablan mal de ti & 16.9 & 15.1 & 9.8 & 6.2 & 2.6 & 2.6 & 53.2 \\
\hline Te insultan & 21.4 & 16.9 & 6.2 & 0.8 & 0.8 & 0.8 & 46.9 \\
\hline Te ofenden & 19.6 & 16.9 & 5.3 & 1.7 & 0.8 & 1.7 & 46.0 \\
\hline Te humillan & 19.6 & 18.7 & 2.6 & 1.7 & 0.8 & 0.8 & 44.2 \\
\hline Se burlan de tu apariencia & 14.2 & 9.8 & 3.5 & 2.6 & 0.8 & 1.7 & 32.6 \\
\hline Te ponen apodos hirientes & 12.5 & 7.1 & 4.4 & 1.7 & 1.7 & 0.8 & 28.2 \\
\hline \multicolumn{8}{|l|}{ Físicas } \\
\hline Te empujan & 19.6 & 15.1 & 7.1 & 1.7 & 1.7 & 0.8 & 46 \\
\hline Te roban materiales & 16.9 & 9.8 & 2.6 & 0.8 & 0.8 & 0.8 & 31.7 \\
\hline Te avientan objetos & 17.8 & 9.8 & 1.7 & 0 & 0 & 0 & 29.3 \\
\hline Te pellizcan & 9.8 & 5.3 & 3.5 & 0 & 1.7 & 1.7 & 22.0 \\
\hline Te rompen materiales & 9.8 & 1.7 & 1.7 & 0 & 0.8 & 1.7 & 15.7 \\
\hline Te patean & 8.9 & 1.7 & 2.6 & 0.8 & 0.8 & 0 & 14.8 \\
\hline Te jalan el pelo & 6.2 & 3.5 & 3.5 & 0.8 & 0 & 0 & 14.0 \\
\hline \multicolumn{8}{|l|}{ Sociales y cibernéticas } \\
\hline Te excluyen & 13.3 & 16.0 & 4.4 & 3.5 & 1.7 & 1.7 & 40.6 \\
\hline Te ofenden en redes sociales & 15.1 & 5.3 & 0 & 0.8 & 0 & 0.8 & 22.0 \\
\hline Sufres agresiones en Facebook & 11.6 & 4.4 & 2.6 & 0 & 0 & 0.8 & 19.4 \\
\hline $\begin{array}{l}\text { Te han divulgado rumores en } \\
\text { redes sociales }\end{array}$ & 7.1 & 3.5 & 0.8 & 1.7 & 0 & 0 & 13.1 \\
\hline
\end{tabular}

Fuente: Elaboración propia obtenida en trabajo de campo, 2015. 
La manifestación verbal fue la segunda en presentarse, coincidiendo con Del Tronco (2013) y Defensor del Pueblo (2007), al considerarla habitual y normal; por lo tanto, es común que estudiantes hablen mal de sus compañeros/as al menos una vez a la semana (hombres $16.9 \%$ y mujeres $15.1 \%$ ), los agresores/as expresan $\mathrm{y}$ difunden rumores negativos, en torno a las víctimas, los cuales no siempre son ciertos, pero lo hacen con la intención de dañar la personalidad y menospreciar a las víctimas ante la comunidad estudiantil y académica, ya sea por venganza o gusto. Además, se insulten (hombres $21.4 \%$ y mujeres $16.9 \%$ ); se ofendan (hombres $19.6 \%$ y mujeres $16.9 \%$ ) y humillen (hombres $19.6 \%$ y mujeres $18.7 \%$ ), los datos indican que los hombres utilizan mayormente la agresión verbal en comparación de las mujeres, concordando con Defensor del Pueblo (2007); esto probablemente se deba a que las mujeres son socializadas con estereotipo de no expresar enojo o violencia "calladitas se ven más bonitas", puede asumirse que se limitan en utilizar las agresiones verbales, en comparación con los hombres cuya construcción genérica, les facilita expresarse verbalmente de forma violenta. Así mismo, un estudio realizado con población escolar de 12 a 15 años, por HidalgoRasmussen e Hidalgo-San Martín (2015), en el estado de Jalisco, México, encontraron que mayormente los hombres fungen como víctimas y acosadores/as.

El alumnado no considera las manifestaciones verbales como agresiones porque están habituados a escuchar tales expresiones; sin embargo, al hacerse constantes dañan a las víctimas, al grado de percibirlas como reales dañando su autoestima, conciben que lo que dicen sus "compañeras/os" es verdad, lo cual es preocupante, máxime que cuando cursan la preparatoria, en edades de 15 a 17 años, construyen su personalidad y pueden hacer inherentes los comentarios y desarrollar una personalidad trastornada con su realidad.

La tercera agresión que se presentó fue directa, es decir, la manifestación física, resaltó te empujan (hombres 19.6\% y mujeres $15.1 \%$ ), te roban materiales (hombres $16.9 \%$ y mujeres $9.8 \%$ ) y te avientan objetos (hombres $17.8 \%$ y mujeres $9.8 \%$ ). Los empujones son vistos como parte del juego, del así nos llevamos, y los hombres lo practican mayormente porque tienen más libertad de ser agresivos, al ser parte de su naturaleza, de acuerdo con las creencias culturales.

El robo no se genera por necesidad de tener materiales, sino para perjudicar a las víctimas, lo que hurtan son útiles escolares como lápices, bolígrafos, libros, libretas, etcétera. En ocasiones, no se comete un robo, ya que al día siguiente o al terminar la clase devuelven el material intacto o maltratado, lo hacen para causar daño económico y emocional (frustración e ira) a las víctimas.

En lo que respecta a aventar objetos, esto se concibe como un juego, generalmente avientan bolas de papel, lápices o cualquier basura con la intención de lastimar a las víctimas, para que no participen en la clase o ridiculizarlas, inicia un compañero/a y le prosigue el resto, hombres o mujeres, provocando desorden en la clase y la probable represión al grupo por parte del docente a cargo; sin embargo, el castigo se basa en el desorden generado no por la agresión que sufre la víctima, de manera que la planta docente juega un papel importante como mitigador/a o perpetuador/a del bullying.

La manifestación social que se presentó fue la exclusión social (16\% de alumnas y $13.3 \%$ de alumnos) coincidiendo con Olweus (2006) y Díaz-Aguado (2005), quienes mencionan que las mujeres están mayormente expuestas al aislamiento de grupos sociales o equipos de trabajo; nadie les habla y no las hacen partícipes de actividades en clase, dinámicas escolares, juegos, etcétera; por lo tanto, se sienten solas y con baja autoestima. La exclusión social se da por venganza, lucha de ego, de belleza o de pareja, las alumnas demuestran su poder aislando a las víctimas de cualquier relación social.

En las manifestaciones cibernéticas $15.1 \%$ de hombres y $5.3 \%$ de mujeres sufren agresiones mediante mensajes ofensivos publicados en su perfil de Facebook, de esa manera gran cantidad de personas miran las ofensas y el daño es mayor. Facebook es el medio más utilizado, porque sólo es necesario conocer el nombre de la persona para dañarla. 


\section{Agresiones que estudiantes han hecho a sus compañeros/as}

Los estudiantes encuestados/as, $21.4 \%$ indicó que ha hecho bullying (hombres $15.1 \%$ y mujeres $6.2 \%$ ); resalta que $78.6 \%$ de la población encuestada expresó no agredir a sus compañeras/os; sin embargo, al preguntarles por alguna manifestación hecha, el porcentaje de agresores/as aumentó, esto muestra que sus acciones no las conciben como bullying, no son consientes de que lastiman física, moral o psicológicamente a sus compañeros/as, quienes por lo general se quedan callados/as. En ese sentido, se coincide con Del Tronco (2013), quien considera que para los acosadores/as las agresiones son un acto de diversión, broma o juego. Los hombres principalmente agreden a sus compañeros/as para demostrar su poder ante la población estudiantil; ya que entre más demuestre su dominio más seguridad tendrán las mujeres de estar a su lado, de acuerdo con los estereotipos de género las mujeres tienen que ser protegidas por los hombres, por lo que serán más codiciados ante las mujeres.

Los acosadores (15.1\%) y las acosadoras (11.6\%) mencionan que la agresión que practican con mayor frecuencia es la manifestación psicológica (Tabla 2), representada en ignorar a sus compañeros/as, lo que coincide con lo que expresaron las víctimas, esta manifestación la hacen con la finalidad de excluir a la persona, en concordancia con Skrzypiec (2008), Olweus (2006) y DíazAguado (2005); en ese sentido, los hombres, principalmente, ubican a las víctimas fuera del grupo social, a un nivel inferior, con la finalidad de demostrar su superioridad, la cual ha sido culturalmente heredada por muchas generaciones.

Tabla 2. Manifestaciones del bullying de acosadores/as.

\begin{tabular}{|c|c|c|c|c|c|c|c|}
\hline \multirow[b]{2}{*}{ Manifestaciones del bullying } & \multicolumn{2}{|c|}{ Una vez a la semana } & \multicolumn{2}{|c|}{ Dos veces a la semana } & \multicolumn{2}{|c|}{ Diario } & \multirow[b]{2}{*}{$\begin{array}{c}\text { Porcentaje } \\
\text { total }\end{array}$} \\
\hline & $\begin{array}{c}\text { Hombres } \\
\%\end{array}$ & $\begin{array}{c}\text { Mujeres } \\
\%\end{array}$ & $\begin{array}{c}\text { Hombres } \\
\%\end{array}$ & $\begin{array}{c}\text { Mujeres } \\
\%\end{array}$ & $\begin{array}{c}\text { Hombres } \\
\%\end{array}$ & $\begin{array}{c}\text { Mujeres } \\
\%\end{array}$ & \\
\hline \multicolumn{8}{|l|}{ Psicológicas } \\
\hline Ignoras & 15.1 & 11.6 & 7.1 & 2.6 & 0.8 & 1.7 & 38.9 \\
\hline No dejas participar en juegos & 5.3 & 2.6 & 0 & 0 & 0 & 0 & 7.9 \\
\hline No dejas participar en clase & 6.2 & 0 & 0.8 & 0 & 0 & 0 & 7.0 \\
\hline \multicolumn{8}{|l|}{ Verbales } \\
\hline Insultas & 16.0 & 8.0 & 4.4 & 2.6 & 0 & 0 & 31 \\
\hline Ofendes & 16.0 & 8.9 & 0 & 2.6 & 0 & 0 & 27.5 \\
\hline Hablas mal de alguien & 11.6 & 10.7 & 0 & 2.6 & 0.8 & 0 & 25.7 \\
\hline Humillas & 11.6 & 5.3 & 0 & 0 & 0 & 0 & 16.9 \\
\hline Haces gestos & 8.0 & 6.2 & 0.8 & 0.8 & 0 & 0 & 15.8 \\
\hline Pones apodos hirientes & 8.9 & 3.5 & 0.8 & 0.8 & 0 & 0 & 14 \\
\hline \multicolumn{8}{|l|}{ Físicas } \\
\hline Empujas & 16.0 & 9.8 & 3.5 & 0.8 & 0.8 & 0 & 30.9 \\
\hline Avientas objetos & 9.8 & 3.5 & 1.7 & 0.8 & 0 & 0 & 15.8 \\
\hline Pellizcas & 5.3 & 3.5 & 1.7 & 0.8 & 1.7 & 0 & 13 \\
\hline Pateas & 8.0 & 2.6 & 1.7 & 0 & 0 & 0 & 12.3 \\
\hline Has roto algún material & 9.8 & 1.7 & 0 & 0 & 0 & 0 & 11.5 \\
\hline \multicolumn{8}{|l|}{ Sociales y cibernéticas } \\
\hline Excluyes & 6.2 & 3.5 & 0 & 0 & 0 & 0 & 9.7 \\
\hline Agredes en WhatsApp & 5.3 & 1.7 & 0 & 0 & 0 & 0 & 7.0 \\
\hline Agredes en Facebook & 5.3 & 0.8 & 0 & 0 & 0 & 0 & 6.1 \\
\hline
\end{tabular}

Fuente: Elaboración propia obtenida en trabajo de campo, 2015.

La segunda manifestación del bullying hecha fue la verbal, no se coincide con Defensor del Pueblo (2007) quien indicó que la manifestación verbal es la más habitual; destacó que al menos una vez a la semana, 16\% de alumnos y $8 \%$ de alumnas han insultado a algún compañero/a, esto coincide con ReyesSánchez, Martínez-Ferrer y Moreno (2015) y Defensor del Pueblo (2007) al expresar que los hombres utilizan mayormente la agresión 


\section{ManifestaCiones del bULLYing en la Preparatoria Agrícola. UNIVERSIDAD AUTÓNOMA DE CHAPINGO, MÉXICO}

verbal en comparación con las mujeres, debido a procesos de socialización de género que enseña a las mujeres ser calladas y obedientes, mientras que los hombres pueden expresarse abiertamente.

La manifestación física, mediante empujones, ocupó el tercer lugar en las agresiones hechas (acosadores 16\% y acosadoras 9.8\%), cuando van caminando empujan al compañero/a con la intención de divertirse o distraerse; si la víctima es pasiva no hará nada y se alejará; en cambio, si es activa responderá a la agresión con el mismo ataque, dando inicio a una pelea, a un intercambio de palabras altisonantes, burlas, o a una disculpa, dependiendo de la personalidad de cada participante.

Es importante destacar que hombres y mujeres hacen bullying a sus compañeros/as, lo observan o son víctimas, lo que coincide con Defensor del Pueblo (2007) y Sullivan et al. (2005), quienes han determinado que el bullying no es exclusivo para un género, ya que alumnas y alumnos están propensos/as a jugar cualquier rol (observador/a, acosador/a o víctima) en el triángulo del bullying; sin embargo, coincidiendo con Reyes-Sánchez et al. (2015) los hombres se involucran principalmente en las diferentes agresiones debido a que, culturalmente, tienen que cumplir con sus roles de agresivos, fuertes, valientes y machos.

\section{Agresiones que observa el alumnado por parte de sus compañeros y compañeras}

El alumnado de la Preparatoria Agrícola, quienes observan las agresiones del bullying, perciben con mayor frecuencia las manifestaciones físicas, en comparación con las víctimas y victimarios/as quienes consideran que las principales manifestaciones del bullying son las agresiones psicológicas. Es decir, los observadores/as manifiestan lo que ven y lo que perciben ser agresiones, en comparación con las víctimas y acosadores y acosadoras quienes manifiestan lo que sienten, de ahí la importancia de considerar las perspectivas de todas las personas involucradas en el triángulo del bullying.

En ese sentido, la Tabla 3 muestra que las y los observadores $(51.5 \%)$ determinan que ven con mayor frecuencia, al menos una vez a la semana, empujones $(24.1 \%$ hombres y $11.6 \%$ mujeres), seguidas por patadas con $47 \%$ (22.3\% hombres y $11.6 \%$ mujeres), lo cual coincide con lo expresado por Olweus (2006) y García (2010), quienes manifiestan que las principales agresiones son dirigidas a causar daño directo a las víctimas, lo cual se hace para demostrar el poder del acosador/a y así ganar respeto o miedo por parte de las víctimas y de los observadores y observadoras. De acuerdo con las y los observadores/as, los hombres usan más esta agresión porque no tienen miedos, la sociedad les ha obligado a ser agresivos y fuertes para demostrar su poder, en cambio a las mujeres les han inculcado el ser para los otros (Lagarde, 1997), sumisas y débiles.

En el caso de las manifestaciones verbales, las agresiones con más alto porcentaje, al menos una vez a la semana, son ofensas $(51.5 \%)$, apodos hirientes (46.2\%) y humillaciones (41.7\%), el resultado coincide con Defensor del Pueblo (2007) en que los hombres agreden más verbalmente en comparación con las mujeres. Los hombres usan más esta agresión porque no tienen miedo en expresar sus pensamientos en público, en cambio a las mujeres les han inculcado el ser calladas, por lo que difícilmente manifiestan sus emociones.

Los acosadores y acosadoras aumentan su autoestima ofendiendo a sus compañeros/as, las ofensas pueden ser desde leves, tonto/a, hasta graves, no sirves para nada, sí escuchan las agresiones constantemente las interiorizarán y dañará el auto-concepto de las víctimas.

En las manifestaciones psicológicas, en tercer lugar, observan como sus compañeros/as son ignorados/as (44.3\%), al menos una vez a la semana ( $15.1 \%$ hombres y $12.5 \%$ mujeres). 
Rosalva Ruiz-Ramírez - CRISTINA SÁnChez-Romero - Emma ZaPata-Martelo - José Luis García-CuÉ Ma. Antonia Pérez-Olvera - Beatriz Martínez-Corona - Gustavo Rojo-Martínez

Tabla 3. Manifestaciones del bullying que observa el alumnado.

\begin{tabular}{|c|c|c|c|c|c|c|c|}
\hline \multirow[b]{2}{*}{ Manifestaciones del bullying } & \multicolumn{2}{|c|}{ Una vez a la semana } & \multicolumn{2}{|c|}{ Dos veces a la semana } & \multicolumn{2}{|c|}{ Diario } & \multirow{2}{*}{$\begin{array}{c}\text { Porcentaje } \\
\text { total }\end{array}$} \\
\hline & $\begin{array}{c}\text { Hombres } \\
\%\end{array}$ & $\begin{array}{c}\text { Mujeres } \\
\%\end{array}$ & Hombres \% & $\begin{array}{c}\text { Mujeres } \\
\%\end{array}$ & $\begin{array}{c}\text { Hombres } \\
\%\end{array}$ & $\begin{array}{c}\text { Mujeres } \\
\%\end{array}$ & \\
\hline \multicolumn{8}{|l|}{ Físicas } \\
\hline Empujones & 24.1 & 11.6 & 4.4 & 7.1 & 3.5 & 0.8 & 51.5 \\
\hline Patadas & 22.3 & 11.6 & 5.3 & 4.4 & 2.6 & 0.8 & 47 \\
\hline Aventar objetos & 14.2 & 9.8 & 6.2 & 1.7 & 1.7 & 0.8 & 34.4 \\
\hline Pellizcar & 11.6 & 5.3 & 6.2 & 1.7 & 1.7 & 0.8 & 27.3 \\
\hline Dar puñetazos & 11.6 & 5.3 & 4.4 & 0.8 & 1.7 & 0.8 & 24.6 \\
\hline Jalar el pelo & 14.2 & 4.4 & 1.7 & 1.7 & 0.8 & 0.8 & 23.6 \\
\hline Robar materiales & 11.6 & 4.4 & 3.5 & 1.7 & 0.8 & 0.8 & 22.8 \\
\hline Romper materiales & 14.2 & 4.4 & 1.7 & 1.7 & 0 & 0.8 & 22.8 \\
\hline \multicolumn{8}{|l|}{ Verbales } \\
\hline Ofender & 18.7 & 14.2 & 11.6 & 6.2 & 0.8 & 0 & 51.5 \\
\hline Poner apodos hirientes & 10.7 & 11.6 & 11.6 & 4.4 & 5.3 & 2.6 & 46.2 \\
\hline Humillar & 14.2 & 11.6 & 8.9 & 5.3 & 1.7 & 0 & 41.7 \\
\hline Contar chistes hirientes & 13.3 & 7.1 & 6.2 & 6.2 & 2.6 & 1.7 & 37.1 \\
\hline $\begin{array}{l}\text { Burlarse por las preferencias } \\
\text { sexuales }\end{array}$ & 8.9 & 6.2 & 7.1 & 6.2 & 6.2 & 0.8 & 35.4 \\
\hline Amenazar verbalmente & 14.2 & 4.4 & 7.1 & 3.5 & 1.7 & 0.8 & 31.7 \\
\hline Burlarse por su origen & 13.3 & 8.0 & 4.4 & 2.6 & 2.6 & 0 & 30.9 \\
\hline \multirow{2}{*}{\multicolumn{8}{|c|}{$\begin{array}{l}\text { Psicológicas } \\
\text { Pulda }\end{array}$}} \\
\hline & & & & & & & \\
\hline Ignorar & 15.1 & 12.5 & 8.9 & 3.5 & 3.5 & 0.8 & 44.3 \\
\hline Hablar mal de alguien & 8.9 & 1.7 & 6.2 & 6.2 & 3.5 & 2.6 & 29.1 \\
\hline Hacer gestos & 11.6 & 5.3 & 2.6 & 1.7 & 3.5 & 2.6 & 27.3 \\
\hline No dejar participar en clase & 9.8 & 8.9 & 4.4 & 2.6 & 0.8 & 0 & 26.5 \\
\hline Difamar & 11.6 & 4.4 & 2.6 & 0.8 & 1.7 & 1.7 & 23.8 \\
\hline \multicolumn{8}{|l|}{ Sociales y cibernéticas } \\
\hline Excluir & 12.5 & 7.1 & 5.3 & 5.3 & 1.7 & 0.8 & 32.7 \\
\hline Agredir en Facebook & 10.7 & 5.3 & 3.5 & 2.6 & 0.8 & 0 & 22.9 \\
\hline $\begin{array}{l}\text { Recibir mensajes ofensivos en } \\
\text { redes sociales }\end{array}$ & 9.8 & 1.7 & 4.4 & 4.4 & 0 & 0 & 20.3 \\
\hline Amenazar en redes sociales & 11.6 & 0.8 & 2.6 & 2.6 & 0 & 0 & 17.6 \\
\hline
\end{tabular}

Fuente: Elaboración propia obtenida en trabajo de campo, 2015.

En la manifestación psicológica, el ignorar $(44.3 \%)$ fue la agresión más común para observadores/as, víctimas o acosadores/ as, coincidiendo con Olweus (2006) quien identificó que esta agresión es la más común de bullying indirecto; hombres y mujeres ignoran a sus compañeros/as provocando que se sientan desvalorizadas/os. Prefieren esta agresión porque no es visible para otras personas de modo que no puede generar algún castigo, además se convierte en peligrosa de acuerdo con Olweus (2006). Las agresiones psicológicas tienen la intención de dañar la autopercepción de las víctimas disminuyendo su valía, quedando susceptibles a cualquier daño o manipulación de los acosadores/as, el cual es el objetivo de los mismos y las mismas.

En las manifestaciones sociales, la exclusión social con $32.7 \%$ (12.5\% observadores y $7.1 \%$ observadoras), fue la más frecuente. En las agresiones cibernéticas la manifestación más alta fue observar agresiones en Facebook (22.9\%), donde observadoras $(5.3 \%)$ y observadores $(10.7 \%)$ han visto que sus compañeros/as son agredidas/os. Facebook se ha convertido en la red social favorita y común para que las acosadoras/es agredan a sus compañeras/os, al ser de fácil acceso y tener poca restricción; además, es mayor la incidencia de daño porque el mensaje llega a miles de usuarios/as de la red social en segundos.

\section{Correlación de las manifestaciones del bullying para las víctimas}

El análisis de correlación es significativo para la variable que indica que las alumnas de la Preparatoria Agrícola son víctimas de violencia 


\section{Manifestaciones del bullying en la Preparatoria Agrícola. UNIVERSIDAD AUTÓNOMA DE CHAPINGO, MÉXICO}

de género, al recibir miradas morbosas de sus compañeros (Tabla 4), lo que coincide con Mingo (2010) y Ruiz-Ramírez y Ayala-Carrillo (2016), debido a los estereotipos de género las mujeres son esencialmente víctimas de violencia de género por el hecho de ser mujeres y ser vistas como objetos sexuales. Existe diferencia significativa en que las mujeres se sienten excluidas de los grupos sociales o de actividades escolares, concordando con Olweus (2006) y Díaz-Aguado (2005), este problema es una forma de violencia de género, ya que el trato que reciben las mujeres es desigual, considerándolas inferiores y por lo tanto no merecen recibir atención y las excluyen.

Tabla 4. Manifestaciones del bullying para las víctimas.

\begin{tabular}{lccc}
\hline & Rho & Pr $>$ F & Significancia \\
Género - Recibes miradas morbosas & 0.341 & 0.000 & $* *$ \\
Género - Te excluyen & 0.219 & 0.020 & $*$ \\
Semestre - Te han difamado & 0.253 & 0.007 & $* *$ \\
Semestre - Te han manoseado sin permiso & 0.201 & 0.034 & $*$ \\
\hline
\end{tabular}

Fuente: Elaboración propia obtenida en trabajo de campo, 2015.

Asimismo, existe significancia en que estudiantes de quinto semestre se han sentido difamados/as y manoseados/as. En la difamación se divulgan rumores negativos hacia las víctimas con el objetivo de que sean vistas como malas personas y así dañar su personalidad y aislarlas del grupo. El manoseo puede ser desde una nalgada hasta un roce, sin querer, en las partes íntimas de las víctimas, con lo cual, el acosador cumple con las reglas de masculinidad al mostrar su virilidad.

\section{Correlación de las manifestaciones del bullying de acosadores y acosadoras}

Los resultados, en la Tabla 5, muestran diferencia significativa en que el estudiantado que pertenece a grupos indígenas ofenden, han roto algún material, han insultado y han hablado mal de sus compañeros y compañeras, en ese sentido, el alumnado indígena han desarrollado medios de defensa ante el bullying, evitan ser las víctimas y se convierten en acosadores/as; por lo tanto, no manifiestan vulnerabilidad ante su condición indígena.

Existe significancia en que las alumnas se burlan de sus compañeros/as por problemas de lenguaje, las víctimas son criticadas por tartamudear o decir palabras incorrectas (dislexia) al momento de realizar lecturas. Los alumnos no dejan participar en clases a sus compañeros/as, al momento de la intervención empiezan a decir que hable otro/a o le avientan objetos para que se calle, provocando que el estudiante no participe en clase y afecte su calificación y autoestima, mientras que el acosador/a aumenta su ego al demostrar dominio en el grupo. 
Tabla 5. Manifestaciones del bullying de acosadores y acosadoras.

\begin{tabular}{lccc}
\hline & Rho & Pr $>$ F & Significancia \\
\hline Grupo indígena - Ofendes & 0.298 & 0.001 & $* *$ \\
Grupo indígena - Rompes materiales & 0.212 & 0.025 & $*$ \\
Grupo indígena - Insultas & 0.199 & 0.035 & $*$ \\
Grupo indígena- Hablas mal de alguien & 0.199 & 0.036 & $*$ \\
Género - Te burlas por problemas de lenguaje & 0.188 & 0.047 & $*$ \\
Género - No dejas participar en clase & -0.219 & 0.020 & $*$ \\
Promedio - Excluyes & 0.207 & 0.029 & $*$ \\
Promedio - Te burlas por problemas de lenguaje & 0.204 & 0.031 & $*$ \\
Promedio - Hablas mal de alguien & 0.189 & 0.046 & $*$ \\
Promedio - Infundes miedo & 0.201 & 0.033 & $*$ \\
Promedio - Agredes en WhatsApp & 0.206 & 0.030 & $*$ \\
\hline
\end{tabular}

Fuente: Elaboración propia obtenida en trabajo de campo, 2015.

La correlación indica que el alumnado con mayor promedio escolar, de primer semestre, hablan mal de sus compañeras/os, las y los excluyen, les infunden miedo y agreden por WhatsApp; en ese sentido, los acosadores/as que se encuentran en esta preparatoria agrícola, de acuerdo con Delgado de los Santos (2015) entran en la clasificación de acosadores/as intelectuales, y no cumplen con el criterio encontrado por Castro (2006) quien menciona que los acosadores/as tienen bajo rendimiento académico, tal vez se deba a que al llegar a la preparatoria tienen que mostrar su fortaleza y dominio para evitar ser novatos/as, y sean las víctimas de las diferentes agresiones. Esta investigación muestra que el estudiantado que pertenece a grupos indígenas o que tienen "promedios de calificación alta," son acosadores/as y no las principales víctimas del bullying.

\section{Correlación de las manifestaciones del bullying de observadores y observadoras}

El análisis de correlación indica diferencia significativa en que el alumnado de quinto semestre son quienes mayormente observan las manifestaciones del bullying en su entorno escolar (Tabla 6), destacan: las agresiones físicas como empujar, jalar el pelo o recibir cachetadas; agresiones verbales como amenazas, humillaciones, insultos, apodos hirientes, burlas por la apariencia física, por vestimenta, problemas de leguaje o por las preferencias sexuales; agresiones psicológicas como ignorar, no dejar participar en clases, recibir notas hirientes o hacer gestos; y agresiones cibernéticas como etiquetar en fotos comprometedoras o recibir mensajes ofensivos en redes sociales. En ese sentido, se destaca que para los observadores/as de quinto semestre no es normal observar que sus compañeros/as sean agredidos/as, tienen más alta la percepción y concientización del bullying, lo que coincide con Ruiz-Ramírez et al. (2016). 


\section{Manifestaciones del bullying en la Preparatoria Agrícola. UnIVERSIDAD AUTÓNOMA DE CHAPINGO, MÉXICO}

Tabla 6. Manifestaciones del bullying de observadores/as.

\begin{tabular}{lccc}
\hline & Rho & Pr $>$ F & Significancia \\
\hline Semestre - Amenazar verbalmente & 0.299 & 0.001 & $* *$ \\
Semestre - Humillar & 0.285 & 0.002 & $* *$ \\
Semestre - Poner apodos & 0.261 & 0.005 & $* *$ \\
Semestre - Burlarse por la apariencia & 0.350 & 0.000 & $* *$ \\
Semestre - Burlarse por la vestimenta & 0.319 & 0.001 & $* *$ \\
Semestre - Burlarse por problemas de lenguaje & 0.277 & 0.003 & $* *$ \\
Semestre - Burlarse por problemas en la vista & 0.302 & 0.001 & $* *$ \\
Semestre - Burlarse por las preferencias sexuales & 0.258 & 0.006 & $* *$ \\
Semestre - Insultar & 0.295 & 0.002 & $* *$ \\
Semestre - Hablar mal de alguien & 0.337 & 0.000 & $* *$ \\
Semestre - Ignorar & 0.288 & 0.002 & $* *$ \\
Semestre - No dejar participar en clase & 0.301 & 0.001 & $* *$ \\
Semestre - Hacer gestos & 0.191 & 0.044 & $* *$ \\
Semestre - Recibir notas hirientes & 0.311 & 0.001 & $* *$ \\
Semestre - Empujar & 0.285 & 0.002 & $*$ \\
Semestre - Jalar el pelo & 0.233 & 0.014 & $*$ \\
Semestre - Dar puñetazos & 0.221 & 0.019 & $*$ \\
Semestre - Recibir cachetadas & 0.357 & 0.199 & $*$ \\
Semestre - Romper materiales & 0.196 & 0.039 & $*$ \\
Semestre - Ofender & 0.190 & 0.045 & $*$ \\
Semestre - infundir miedo & 0.225 & 0.017 & $*$ \\
Semestre - Excluir & 0.205 & 0.030 & $*$ \\
Semestre - Obligar a hacer cosas & 0.196 & 0.038 & $*$ \\
Semestre - Recibir miradas morbosas & 0.210 & 0.026 & $*$ \\
\hline
\end{tabular}

Fuente: Elaboración propia obtenida en trabajo de campo, 2015.

\section{Conclusiones}

Con base en los resultados obtenidos, el objetivo de investigación se cumplió al analizar las manifestaciones del bullying en estudiantes de una Preparatoria Agrícola de México; el supuesto no se rechazó. En ese sentido, las principales manifestaciones del bullying en la Preparatoria Agrícola son: en las víctimas y los acosadores/as son las psicológicas, mediante el ignorar. En cambio, las observadoras/es identifican que la principal agresión que miran son las manifestaciones físicas con empujones.

En ese sentido, se destaca que las personas involucradas en eltriángulo del bullying, víctima, acosador/a y observador/a, tienen diferentes representaciones de las manifestaciones del bullying. Por lo tanto, se sugiere que las futuras investigaciones relacionadas con bullying no generalicen sus manifestaciones, deben considerar las diferentes perspectivas de los actores y actrices, ya que la percepción y el sentimiento ante las agresiones son diferentes en cada uno de ellos y ellas; así mismo, es importante que determinen específicamente los tipos de agresiones físicas, psicológicas, verbales, entre otras, que se reciben, hacen $u$ observan, para ver holísticamente el problema del bullying y se puedan diseñar estrategias, más eficaces, para su prevención y mitigación.

Las manifestaciones del bullying no son exclusivas de las mujeres o de los hombres, ambos géneros asumen distintos roles en el triángulo del bullying, ya sea como víctima, acosador/a u observador/a; sin embargo, en está investigación fueron los hombres, quienes, en cualquiera de los roles, tienen mayor participación.

Las correlaciones indicaron que estudiantes con calificaciones altas o quienes pertenecen a grupos indígenas son acosadores/as, y no son las principales víctimas del bullying, en contraste 
con diferentes investigaciones realizadas al respecto. En ese sentido, sobresale que el alumnado indígena ha desarrollado estrategias de defensa para evitar que sean las principales víctimas del bullying, se han convertido en acosadores/as; ofenden, rompen materiales, insultan y hablan mal de sus compañeros/as; por lo tanto, han aprendido a no manifestar vulnerabilidad por su origen étnico.

Alumnas y alumnos de quinto semestre mayormente observan las manifestaciones del bullying en su entorno escolar, lo cual se adjudica a que son más conscientes del problema.

\section{Lista de referencias}

Brunstein, A.; Marrocco, F.; Kleiman, M.; Schonfeld I. \& Gould, M. (2007). Bullying, depression, and suicidality in adolescents. J Am Acad Child Adolesc Psychiatry, 46 (1), pp. 40-49.

Castro, A. (2006). Violencia silenciosa en la escuela. Dinámica del acoso escolar y laboral. Buenos Aires: Bonum.

Defensor del Pueblo (2007). Violencia escolar: el maltrato entre iguales en la educación secundaria obligatoria 1999-2006. Madrid: Publicaciones de la Oficina del Defensor del Pueblo.

Delgado de los Santos, S. (2015). Bullying en la Escuela Secundaria 22 de Septiembre de la Ciudad de Lerdo, Durango. Revista Iberoamericana para la Investigación y el Desarrollo Educativo, 10, pp. 1-10. Recuperado de: http://ride.org.mx/1-11/ index.php/RIDESECUNDARIO/article/ viewFile/373/365

Del Tronco, J. (2013). La violencia en las escuelas secundarias de México. Una exploración de sus dimensiones. México, D. F.: Flacso.

Díaz-Aguado, M. (2005). La violencia entre iguales en la adolescencia y su prevención desde la escuela. Psicothema, 17 (4), pp. 549-558.

García, M. (2010). Violencia en las escuelas. Un relevamiento desde la mirada de los alumnos. Buenos Aires: Ministerio de Educación.
García-Villanueva, J.; De la Rosa-Acosta, A. \& Castillo-Valdés, J. S. (2012). Violencia: análisis de su conceptualización en jóvenes estudiantes de bachillerato. Revista Latinoamericana de Ciencias Sociales, Niñez y Juventud, 10 (1), pp. 495-512.

Ghiso, A. M. (2012). Algunos límites de las respuestas frente a la violencia y la inseguridad, en las instituciones educativas. Revista Latinoamericana de Ciencias Sociales, Niñez y Juventud, 10 (2), pp. 815 824.

Hidalgo-Rasmussen, C. \& Hidalgo-San Martín, A. (2015). Violencia e inseguridad contextual percibida y roles en bullying en escolares mexicanos. Revista Latinoamericana de Ciencias Sociales, Niñez y Juventud, 13 (2), pp. 767-779. Doi :10.11600/1692715x.13215021214.

Lagarde, M. (1997). Género y femenino. Desarrollo humano y democracia. Madrid: Horas y Horas.

Mendoza, B. (2012). Bullying. Los múltiples rostros del acoso escolar. México, D. F.: Pax.

Mingo, A. (2010). Hilvanes de género en la experiencia escolar. En A. Mingo (coord.) Desasosiegos. Relaciones de género en la educación, (pp. 153-198). México, D. F.: Plaza y Valdés.

Olweus, D. (1999). Sweden. En P. K. Smith, J. Morita, T. Junger, D. Olweus, R. Catalano $\&$ P. Slee (eds.) The nature of school acoso escolar: a cross national perspective, (pp 7-27). London: Routledge.

Olweus, D. (2006). Una revisión general. En R. Serrano (coord.) Acoso y violencia en la escuela: cómo detectar, prevenir y resolver el bullying, (pp. 79-106). Barcelona: Ariel.

Onetto, F. (2004). Clima institucional $\mathrm{y}$ pronósticos de violencia. Revista Novedades Educativas, 16 (163), pp. 3437.

Ortega-Rivera, J.; Jiménez, V. \& Ruiz, R. (2010). Violencia sexual y cortejo juvenil. En R. Ortega (ed.) Agresividad injustificada, bullying y violencia escolar, (pp. 211-232). Madrid: Alianza.

Reyes-Sánchez, C.; Martínez-Ferrer, B. \& Moreno, D. (2015). Adolescentes 


\section{ManifestaCiones del bullying en la Preparatoria Agrícola. UnIVERSIDAD AUTÓNOMA DE CHAPINGO, MÉXICO}

agresores en la escuela. Un análisis desde la perspectiva de género. Feminismo/s, 25, pp. 11-131.

Ruiz-Ramírez, R. \& Ayala-Carrillo, R. (2016). Violencia de género en instituciones de educación. Ra Ximhai, 12 (1), pp. 21-32.

Ruiz-Ramírez, R.; Zapata-Martelo, E.; GarcíaCué, J. L.; Pérez-Olvera, Ma. A.; MartínezCorona, B. \& Rojo-Martínez, G. (2016). Bullying en una universidad agrícola del estado de México. Ra Ximhai, 12 (1), pp. 105-126.

Secretaría de Educación Pública-SEP (2014). Encuesta Nacional sobre Exclusión, Intolerancia y Violencia en el Nivel Medio Superior. Primer reporte temático. Recuperado de: http://www.sems.gob.mx/work/models/ sems/Resource/11599/5/images/sems encuesta_violencia_reporte_130621_final. pdf

Secretaría de Seguridad Pública-SSP \& Secretaría de Educación Pública-SEP (2007). Guía básica de prevención de la violencia en el ámbito escolar. Recuperado de: http://www.google.com/url?sa=t\&rct=j $\& \mathrm{q}=\&$ esrc $=$ s\& source $=$ web $\& \mathrm{~cd}=1 \& \mathrm{ved}=$ 0CB8QFjAA\&url=http $\% 3 \mathrm{~A} \% 2 \mathrm{~F} \% 2 \mathrm{Fbas}$ ica.sep.gob.mx $\% 2$ Fescuelasegura $\% 2 \mathrm{Fpdf}$ $\% 2$ FMatInfo\%2FViolenciaEscolar.pdf\&ei $=\mathrm{Z} 6 \mathrm{HjVNGJHYHXggSnsoTgCg \& usg}=\mathrm{A}$ FQjCNEZUTn60z2BZe1m1dDIZOufh4Q 9fQ\&sig2=-E8TioHN6wpoEbx6rrt6fg

Skrzypiec, G. (2008). Living and Learning at School, document presented at the Annual Conference of the Australian Association for Research in Education. Recuperado de: www.aare.edu. au/08pap/skr081125.pdf

Smith, P.; Rigby, K. \& Pepler, D. (2004). Working to prevent school bullying: key issues. En P. Smith; D. Pepler \& K. Rigby (eds.) Bullying in Schools: How Successful Can Interventions Be?, (pp. 1-12). Cambridge: Cambridge University.

Subsecretaría de Educación Media SuperiorSems (2014). Encuesta Nacional sobre Exclusión, Intolerancia y Violencia en Escuelas de Educación Media Superior. México, D. F.: Sems. Recuperado de: http://www.sems.gob.mx/work/models/
sems/Resource/11599/5/images/sems encuesta_violencia_reporte_130621_final. pdf

Sullivan, K.; Cleary, M. \& Sullivan, G. (2005). Bullying en la enseñanza secundaria. El acoso escolar: cómo se presenta y cómo afrontarlo. Barcelona: Ceac.

Tamar, F. (2005). Maltrato entre escolares (bullying): estrategias de manejo que implementan los profesores al interior del establecimiento escolar. Psyckhe, 14 (1), pp. 211-225.

Varhama, L. \& Björkqvist, K. (2005). Relation between school bullying during adolescence and subsequent long-term unemployment in adult hood in a Finnish sample. Psychol Rep, 96 (2), pp. 269-272.

Voors, W. (2005). Bullying el Acoso Escolar. Barcelona: Oniro, S. A.

Zapata-Martelo, E. \& Ruiz-Ramírez, R. (2015). Respuestas institucionales ante la violencia escolar. Ra ximhai, 11 (4), pp. 475-491. 